\title{
A COMPARISON ON HYPONYMY AND CULTURAL SYMBOLISM OF SIX COLOR WORDS BETWEEN CHINESE LANGUAGE AND BAHASA INDONESIA
}

\author{
汉印尼语六大颜色词的下位词与文化象征意义对比 \\ Kristina Indah Setyo Rahayu
}

Program D3 Bahasa China Fakultas Ilmu Budaya, Universitas Sebelas Maret

Jln. Ir. Sutami 36a Kentingan Surakarta 57126

kristinaindah@hotmail.com

\begin{abstract}
Color words originate from natural world. In general different languages have similar patterns of color hypernymy. Nevertheless, when it comes to color hyponymy, it is not the case. Specific color hyponyms have emerged because different countries have different cultures. Besides, color words are also rich in cultural symbolism. In other words, same colors in different countries have different cultural symbolisms. This paper analyzed six color words (red, white, yellow, blue, green, and black) between Chinese language and bahasa Indonesia to find similarities and differences both in hyponymy and in culture symbolism.
\end{abstract}

Keywords: Chinese language, bahasa Indonesia, colour word, Hyponym, culture symbolism

内容摘要

颜色词源于自然界，各种语言对于颜色上位词有相似的概念。而对于颜色的下位词却不是如此。出现 颜色下位词不同与每个国家的环境有关。另外, 颜色词还包含有不同的文化象征意义。换句话说, 同样的颜色 词在不同的国家有不同的文化象征意义。本文对汉语、印尼语中红、白、黄、蓝、绿、黑六大颜色词的下位词 和文化象征意义进行对比，分析、比较其中的异同。

关键词：汉语, 印尼语, 颜色词, 下位词, 文化象征意义 
汉语颜色词从古代汉语至现代汉语一直受到学 者的重视。关于汉语颜色词发表的文章或写的论文甚 至书也是五花八门。进行汉语颜色词与其他语言对比 研究也不少。尤其是对汉英颜色词的对比。因为英语 毕竟是国际的第一语言, 同时代表了西方国家的语 言。

学者们在进行汉英颜色词对比研究时, 不仅获 得汉语作为中国的语言与英语作为西方国家的语言相 同和相反的意思。而且, 还了解到中西文化的区别。

研究内容、方法

本文研究的主要内容是就汉印尼 “红”、“ 黄”、“蓝”、“绿”、“白” 和 “黑” 六大颜色词 的下位词与文化象征意义进行对比。

通过比较研究, 分析汉印颜色词及其背后文化 的异同。虽目前已有了许多汉语颜色词和别的语言对 比研究的成果, 笔者想本文能够为颜色词研究方面提 供一个补充。

本文主要内容是对比汉语和印尼语中的六个颜 色: “红”、“黄”、“蓝”、“白”、“黑” “ 绿”, 选择这六个颜色原因是因为, 前五个是现代汉 语纯色基本颜色。那么, “绿” 是现代人们所说 “安

\begin{tabular}{|c|c|c|c|c|c|}
\hline No & 词目 & 词典释义 & No & 词目 & 词典释义 \\
\hline 1 & 赤红 & 红 & 8 & 嫩红 & $\begin{array}{l}\text { 像初开杏花那样的 } \\
\text { 浅红色 }\end{array}$ \\
\hline 2 & 大红 & 很红的颜色 & 9 & 浅红 & 淡红 \\
\hline 3 & 橙红 & $\begin{array}{l}\text { 像橙子那样红里带 } \\
\text { 黄的颜色 }\end{array}$ & 10 & 深红 & 浓红 \\
\hline 4 & 绯红 & 鲜红 & 11 & 血红 & 像鲜血那样的红色 \\
\hline 5 & 粉红 & 红和白合成的颜色 & 12 & 红粀形 & 很红 \\
\hline 6 & 红润 & 红而滋润 & 13 & 红艳艳 & 红的鲜邦夺目 \\
\hline 7 & 鲜红 & 形容颜色红而鲜艳 & & & \\
\hline
\end{tabular}
全” 颜色, 认为也值得研究。

本文的写作，主要是通过理论分析、对比研究 两种语言的特点。笔者使用了《现代汉语词典》第五 版, 为参考汉语中的颜色词语的主要参考文献。同时 也使用了《印尼语大词典》 (KAMUS BESAR BAHASA INDONESIA）, 为参考印尼语中的颜色词语的主要参 考文献。另外, 文中有些资料来自: http://www. wikipedia. org/ (维基百科网), 文中不再具体标 出。

\section{研究内容、方法}

环境、习俗对颜色词的下位词具有很大的影 响, 即引起了不同颜色词的下位词。颜色词的下位词 表达了语言的特点与某个地方的文化。此外, 由于颜 色是源于自然界, 同时在不同语言也会出现相同颜色 词的下位词。

1. 红

$$
\text { 汉语的 “红”，印尼语是 “MERAH”。 }
$$

\section{红的概念}

《现代汉语词典》第五版指出, “红” 是指像 鲜血的颜色; 象征喜庆的红布; 象征顺利、成功或受 人重视、欢迎; 象征革命或政治觉悟高; 红利; 姓。

《印尼语大词典》指出, “红” 是指像鲜血. 的颜色或者含有鲜血的颜色。可见, 在汉语与印尼 语中, 同样使用 “血” 来形容红色。另外, 印尼语 中, “红” 的义项没有汉语多。

表2 印尼语中红的下位词

\begin{tabular}{|c|c|c|c|c|c|}
\hline NO & 词目 & 词典释义 & NO & 词目 & 词典释义 \\
\hline 1 & Merah bata & 像砖头的 & 8 & $\begin{array}{l}\text { Merah } \\
\text { lembayung }\end{array}$ & $\begin{array}{l}\text { 像初开杏花那 } \\
\text { 样的浅红色 }\end{array}$ \\
\hline 2 & Merah bungur & 带紫色的 & 9 & Merah marak & 淡红 \\
\hline 3 & Merah dadu & 粉红色的 & 10 & Merah masak & 浓红 \\
\hline 4 & Merah darah & 血红 & 11 & Merah merang & $\begin{array}{l}\text { 像鲜血那样的 } \\
\text { 红色 }\end{array}$ \\
\hline 5 & Merah delima & 像石榴的 & 12 & Merah muda & 很红 \\
\hline 6 & Merah hati & 像心脏的 & 13 & Merah murup & 红的鲜艳夺目 \\
\hline 7 & Merah jambu & 像番樱桃的 & 14 & Merah segar & \\
\hline
\end{tabular}

汉语与印尼语中, “红” 的下位词都比较丰 富。印尼语的“merah segar”、“merah darah” 与汉语的 “血红”、“鲜红”、“绯红” 完全对 应。“merah masak”、“merah merang” 与 “红 润”、“赤红”相同。

表示深红，除了 “merah tua” 和 “深红” 以 外, 印尼语还有 “merah hati”、“merah bata”。 而且, 汉语有 “红删媭”、“红艳艳”。

汉语的 “大红”与印尼语的 “merah murup” 、“merah marak” 相互对应。颜色比较浅的红 色, 印尼语有 “merah dadu”、“merah delima” 、“merah jambu”、“merah muda”, 汉语有 “粉 红”、“浅红” 和 “嫩红” 为相对应。

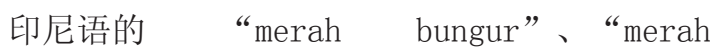
lembayung” , 也是 “红” 颜色的一种。这种颜色在 汉语 “红” 颜色中没有相当的对应。汉语的 “橙红” 在印尼语 “红” 颜色词汇没有相当的对应。

汉语中像 “红扑扑、红泮泮、红艳艳” 等词 语, 印尼语中也能用翻译成别的说法, 就是 “红” 词前面加 “ke-”, 后面加 “- an”, 成 “kemerahmerahan” (好像红似的), 如:

(1) Di senja hari pegunungan di ufuk barat kelihatan menjadi biru kemerah-merahan. (傍晚时, 在西边 的山上看起来蓝红殁彤的。)

(2) Dia gadis yang sangat pemalu,sekali mendapat pujian mukanya menjadi kemerah-merahan. (她是个害羞 的姑娘, 一说她好听的话, 一下子就红扑扑的）

“merah bata（像砖头的）”是印尼语 “红” 颜色的下位词比较特别。砖头是印尼人建筑主要 
的东西。这在汉语没有, 因为中国人的建筑几乎不用 砖头的。

2. 白

印尼语 “PUTIH” 是汉语 “白” 的意思。汉语 有 “红白喜事” , 告诉了人们 “红” 和 “白” 颜 色对中国人来说具有很强的重要性。作为国旗的 颜色, 红白色对印尼人来说这两种颜色是很神圣 的颜色。

\section{白的概念}

据《现代汉语词典》第五版，“白”颜色的 意思是 1. (形) 像霜或雪的颜色 (跟 “黑” 相对) 。2. (形) 光亮; 明亮。3. 清楚; 明白; 弄明白。 4 . 没有加上什么东西的; 空白。5. 象征反动。因为 “ 白” 在汉语有几种意思和意义, 其中不是 “白” 颜色 的意思。所以，笔者只调几个意思。

据《印尼语大词典》里, 印尼语 “白”颜色意 思是 1 . 像棉花的颜色。2. (形) 纯洁; 清白。3. ( 形）苍白。

表3 汉语中白的下位词

\begin{tabular}{|c|c|c|c|c|c|}
\hline NO & 词目 & 词典释义 & No & 词目 & 词典释义 \\
\hline 1 & 粹白 & 纯粹; 纯白 & 5 & 白茫茫 & $\begin{array}{l}\text { 形容一望无边的白 } \\
\text { (云、雾、雪、大 } \\
\text { 水等) }\end{array}$ \\
\hline 2 & 白㿠㿠 & $\begin{array}{l}\text { 形容霜、雪等 } \\
\text { 洁 }\end{array}$ & 6 & 白蒙蒙 & $\begin{array}{l}\text { 形容烟、雾、蒸气 } \\
\text { 等白茫茫一片, 模 } \\
\text { 糊不清。 }\end{array}$ \\
\hline 3 & 白花花 & 白得耀眼 & 7 & 乳白 & 像奶汁的颜色 \\
\hline 4 & 白晃晃 & 白而亮 & 8 & 雪白 & 像雪那样的洁白 \\
\hline
\end{tabular}

表4 印尼语中白的下位词

\begin{tabular}{clc}
\hline N0 & \multicolumn{1}{c}{ 词目 } & 词典释义 \\
\hline 1 & Putih bersih & 洁白 \\
2 & Putih kuning & 淡黄 \\
3 & Putih meta / metah & 非常白 \\
4 & Putih kapas & 像棉花白 \\
5 & Putih susu & 奶汁白 \\
\hline
\end{tabular}

“白” 颜色是无任何颜色, 也是说素色。汉语 表示白色使用了霜、雪、雾等, 真正的表达了白色是 什么样的颜色。印尼语白色主要是表示纯洁、洁净的 意思, 最多是用像奶的颜色来形容, 所以白颜色词汇 很少用到其他事物来形容它。

印尼语的 “putih bersih” 与汉语的 “粹白” 相同对应。汉语中与印尼语的 “putih meta/metah” 相对的有 “白花花”、“白光光”。至于 “putih susu” 就与 “乳白” 相同的语义特征。

“白皑皑”、“白茫茫”、“白蒙蒙”, “ 雪白”, 在印尼语里没有相对应的, 印尼语中只能 称 “putih” 或相近的短语翻译。

“putih kapas（棉花白）”, 是印尼语中最 有代表 “白” 颜色的下位词。因为棉花是织布的主要 原料。印尼的五项原则的第五个是说 “印尼人民的社 会正义”，使用棉花和稻子来象征, 意思就是希望人 民过得好。
3. 黄

黄色, 印尼语是KUNING。

\section{黄的概念}

《现代汉语词典》第5版，指出 “黄” 是指 1. (形) 像丝瓜花或向日葵花的颜色。2. 指黄金。3. 指蛋黄。4. (形) 象征腐化芏落, 特指色情。

《印尼语大词典》指出, “黄”是指像姜黄或 黄金的颜色。

表5 汉语中黄的下位词

\begin{tabular}{|c|c|c|c|c|c|}
\hline NO & 词目 & 词典释义 & NO & 词目 & 词典释义 \\
\hline 1. & 黄灿灿 & $\begin{array}{l}\text { 形容金黄而 } \\
\text { 鲜脃 }\end{array}$ & 7. & 姜黄 & $\begin{array}{l}\text { 形容像姜那样的 } \\
\text { 黄色 }\end{array}$ \\
\hline 2. & 黄澄澄 & 形容金黄色 & 8. & 嫩黄 & $\begin{array}{l}\text { 像非黄那样的浅 } \\
\text { 黄色 }\end{array}$ \\
\hline 3. & 鹅黄 & $\begin{array}{l}\text { 像小鹅线毛那 } \\
\text { 样的黄色; } \\
\text { 嫩黄 }\end{array}$ & 9. & 蛋黄色 & 像鸡蛋黄的 \\
\hline 4. & 金黄 & $\begin{array}{l}\text { 黄而微红略像 } \\
\text { 金子的颜色 }\end{array}$ & 10. & 米黄 & 白而微黄的颜色 \\
\hline 5. & 橘黄 & $\begin{array}{l}\text { 比黄色略深像 } \\
\text { 橘子皮的颜色 }\end{array}$ & 11. & 乳黄 & $\begin{array}{l}\text { 像奶油那样的淡 } \\
\text { 黄色 }\end{array}$ \\
\hline 6. & 橙黄 & $\begin{array}{l}\text { 像橙子那样黄 } \\
\text { 里带红的颜色 }\end{array}$ & & & \\
\hline
\end{tabular}

表6 印尼语中黄的下位词

\begin{tabular}{clc}
\hline N0 & \multicolumn{1}{c}{ 词目 } & 词典释义 \\
\hline 1 & Kuning emas & 金黄色 \\
2 & Kuning ke-emasan & 黄灿灿 \\
3 & Kuning gading & 淡黄色, 米黄色 \\
4 & Kuning telur & 蛋黄 \\
5 & Putih kuning & 淡黄 \\
\hline
\end{tabular}

印尼语的 “黄” 颜色的语义场没有汉语多。印 尼语的 “kuning emas”与汉语的 “金黄”、“黄澄 澄” 对应。印尼语的 “kuning ke-emasan” 与汉语 的 “黄灿灿” 对应，都有 [发亮的金黄] 语义特征。 这表示在两种语言之间都认为黄色为 “贵色” , 因为 黄金是贵重的东西。所以, “金黄”、“黄灿灿”之 类的下位词有相同的概念。

印尼语的 “kuning gading”、“putih kuning”与汉语的 “米黄”、“乳黄” 相互对应。印 尼语的 “kuning telur” 与汉语的 “蛋黄色” 对应, 都有 [像黄鸡蛋的]语义特征。

虽然印尼语 “黄” 颜色的概念是指像姜黄的颜 色，但在下位词中没有 “姜黄” 的颜色。至于, 汉语 的 “鹅黄”、“姜黄”、“嫩黄”，印尼语只能称 kuning或近似的词语翻译。

4. 蓝

印尼语的 “BIRU” 是指汉语 “蓝”颜色的意思。 蓝的概念

对于蓝色这个颜色, 汉语和印尼语有同样的概 念, 是像晴天空的颜色。 


\begin{tabular}{|c|c|c|c|c|c|}
\hline NO & 词目 & 词典释义 & NO & 词目 & 词典释义 \\
\hline 1. & 宝蓝 & 鲜亮的蓝色 & 7. & 湖蓝 & 浅蓝 \\
\hline 2. & 碧蓝 & 青蓝色 & 8. & 蔚蓝 & $\begin{array}{l}\text { 像晴朗的天空的 } \\
\text { 颜色 }\end{array}$ \\
\hline 3. & 静青 & 深蓝 & 9. & 海蓝 & $\begin{array}{l}\text { 像大海那样的蓝 } \\
\text { 颜色 }\end{array}$ \\
\hline 4. & 蓝晶晶 & $\begin{array}{l}\text { 蓝而发亮 } \\
\text { (水、宝石) }\end{array}$ & 10. & 湛蓝 & $\begin{array}{l}\text { 深蓝色（天空、 } \\
\text { 湖海） }\end{array}$ \\
\hline 5. & $\begin{array}{l}\text { 蓝盈盈、 } \\
\text { 蓝葷 }\end{array}$ & $\begin{array}{l}\text { 蓝得发亮 } \\
\text { (天空) }\end{array}$ & 11. & 浅蓝 & 淡蓝 \\
\hline 6. & 天蓝 & 像晴朗的天空颜色 & & & \\
\hline
\end{tabular}

\begin{tabular}{clc}
\hline NO & \multicolumn{1}{c}{ 词目 } & 词典释义 \\
\hline 1 & Hijau gadung & 浅绿 \\
2 & Hijau lumut & 墨绿 \\
3 & Hijau maya-maya & 鲜绿 \\
4 & Hijau muda & 浅嫩绿 \\
5 & Hijau tua & 深绿 \\
\hline
\end{tabular}

表8 印尼语中蓝的下位词

\begin{tabular}{clc}
\hline N0 & & 词目 \\
\hline 1 & Biru gerau & 词典释义 \\
2 & Biru langit & 带黑色 \\
3 & Biru laut & 天蓝 \\
4 & Biru muda & 海蓝 \\
5 & Biru tua & 浅蓝 \\
\hline
\end{tabular}

印尼语中使用一个词来代表天空的蓝, 就是 “biru langit”，而汉语中使用了几种说法是，“ 天蓝”、“蓝盈盈”、“蓝莹莹”、“蔚蓝”, 还 有 “湛蓝” 也是蓝色天空的意思。只是 “湛蓝” 比 “ 天蓝”稍微深一些。

印尼语的 “biru laut” 与汉语的 “海蓝” “湖蓝”、“湛蓝”相互对应。汉语的“宝蓝”

“碧蓝”、“蓝晶晶”，印尼语中没有相对应的。 印尼语的 “biru tua” 和 “biru muda” ，只是指 “ 浅蓝” 和 “深蓝” 的意思, 而没有具体的相对事物来 代表。“biru gerau” 是比深蓝色稍微深一些。

5. 绿

HIJAU是印尼语的 “绿”。绿色与草木、树叶, 还有自然和环保连在一起。

绿的概念

《现代汉语词典》第5版指出, “绿” 颜色是 指像草和树叶茂盛时的颜色, 有蓝和黄而成。《印尼 语大词典》指像树叶的颜色。

表9 汉语中绿的下位词

\begin{tabular}{|c|c|c|c|c|c|}
\hline NO & 词目 & 词典释义 & NO & 词目 & 词典释义 \\
\hline 1. & 碧绿 & 青绿色 & 9. & 豆绿 & $\begin{array}{l}\text { 像青豆那样的 } \\
\text { 绿色 }\end{array}$ \\
\hline 2. & 翠绿 & $\begin{array}{l}\text { 像翡翠那样的 } \\
\text { 绿色 }\end{array}$ & 10. & 苹果绿 & 浅绿色 \\
\hline 3. & 苍翠 & 深绿（草木等） & 11. & 深绿 & 浓绿 \\
\hline 4. & 浅绿 & 淡绿 & 12. & 橄榄绿 & $\begin{array}{l}\text { 像橄榄果实那样 } \\
\text { 的青绿色 }\end{array}$ \\
\hline 5. & 湖绿 & 淡绿 & 13. & 绿茸茸 & 形容碧绿而稠密 \\
\hline 6. & 草绿 & 绿而略黄的颜色 & 14. & 绿生生 & 形容碧绿而鲜嫩 \\
\hline 7. & 黛绿 & 墨绿 & 15. & 绿芗芗 & 形容晶莹碧绿 \\
\hline 8. & 䓤绿 & $\begin{array}{l}\text { 浅绿而微黄的 } \\
\text { 颜色 }\end{array}$ & 16. & 绿油油 & 形容浓绿而润泽 \\
\hline
\end{tabular}

表11 汉语中黑的下位词

\begin{tabular}{|c|c|c|c|c|c|}
\hline NO & 词目 & 词典释义 & No & 词目 & 词典释义 \\
\hline 1. & 乌黑 & 深黑色 & 6. & 黑蒙蒙 & $\begin{array}{l}\text { 形容光线昏暗, } \\
\text { 看不清楚 }\end{array}$ \\
\hline 2. & 墨黑 & 非常黑; 很暗 & 7. & 黑黢黢 & 形容很黑 \\
\hline 3. & 漆黑 & 非常黑; 很暗 & 8. & 黑魀魃 & 形容黑暗 \\
\hline 4. & 黑洞洞 & 形容黑暗 & 9. & 黑油油 & 形容黑得发亮 \\
\hline 5. & 黑糊糊 & 形容颜色发黑 & 10. & 乌油油 & 形容黑而润泽 \\
\hline
\end{tabular}

印尼语的 “hi jau gadung” 与汉语的 “草绿” “碧绿”、“豆绿”、“翠绿”相对应，只是浓 度有差异。“hijau lumut”、“hijau tua”与 “ 黛绿”、“苍翠”、“深绿” 完全有相互对应。至 于 “hi jau muda” 是与 “浅绿”、“苹果绿” 相同。

汉语中的 “橄榄绿”、“绿茸茸”、“绿生 生”、“绿荣䒯”、“绿油油”, 印尼语的 “hijau maya-maya” 为这些颜色的对应。“苟绿” 印尼语, 只能称为 “hijau”。

6. 黑

“黑”, 印尼语是 “HITAM”。“黑” 颜色作为 最深的颜色, 引起了种种的意义与概念。形容 “ 黑”颜色的食物也是五彩缤纷。

黑的概念

“黑” 的意思是：1. (形) 像煤或墨的颜色 （跟 “白” 相对）。2. (形) 黑暗。3. (名) 夜晚; 黑夜。4. 秘密; 非法的; 不公开的。5. (形) 坏; 狠毒。印尼语意思是像木炭的颜色。

\section{表12 印尼语中黑的下位词}

\begin{tabular}{cccccc}
\hline N0 & 词目 & 词典释义 & N0 & \multicolumn{1}{c}{ 词目 } & \multicolumn{1}{c}{ 词典释义 } \\
\hline 1. & $\begin{array}{l}\text { Hitam } \\
\text { berkilat }\end{array}$ & 黑油油 & 5. & $\begin{array}{l}\text { Hitam } \\
\text { legam }\end{array}$ & 乌黑; 漆黑 \\
2. & $\begin{array}{l}\text { Hitam } \\
\text { birat }\end{array}$ & $\begin{array}{l}\text { 又黑又丑 } \\
\text { 的人 }\end{array}$ & 6. & $\begin{array}{l}\text { Hitam } \\
\text { kumbang }\end{array}$ & 乌黑; 漆黑 \\
3. & $\begin{array}{l}\text { Hitam } \\
\text { jengat }\end{array}$ & 乌黑; 漆黑 & 7. & $\begin{array}{l}\text { Hitam } \\
\text { manggis }\end{array}$ & $\begin{array}{l}\text { 紫黑色 (像山竹 } \\
\text { 的颜色) }\end{array}$ \\
4. & $\begin{array}{l}\text { Hitam } \\
\text { lotong }\end{array}$ & 乌黑; 漆黑 & 8. & $\begin{array}{l}\text { Hitam } \\
\text { pekat }\end{array}$ & 乌黑; 漆黑 \\
\hline
\end{tabular}


汉语和印尼语的 “黑” 的下位词, 都表示 “ 黑暗、黯然” 的颜色。印尼语的 “hitam jengat” 、“hitam lotong”、“hitam legam”、“hitam kumbang”、“hitam pekat” 与汉语的 “乌黑”、“ 墨黑”、“漆黑”、“黑洞洞”、“黑咕隆咚”、“ 黑黢黢”、“黑鬼鬼”, 都有相同的语义特征。

印尼语是 “hitam berkilat”, 汉语有 “黑 油油” 和 “乌油油” 相同的意思。而, 黑蒙蒙 [模 糊的], 印尼语也没有相同的意思。另外, 印尼语 的 “hitam birat” 与汉语的 “黯黑”, 不同的语义 特征。

\section{文化象征意义对比}

语言与文化有很密切的关系。我们用语言表现 出文化, 同时语言也是文化的一部分。语言中的词汇 有多种来源, 其中之一是颜色。那些包含颜色语素 的词却不表示色彩概念的词语, 往往那些词语的 “所 指” 千差万别, 如红人、白眼、绿豆、黑店等等。 含颜色语素的词语不是反映对象的特点, 而是指构词 上 “含颜色”。

关于颜色与文化的联想意义, 叶军在 《现代汉 语色彩词研究》指出, 含颜色词可以归纳出两种情 况, 一种是色彩语素直接参与含色彩词语的意义构 成, 揭示含色彩词语所指事物的色彩特征。如: 绿 豆、黑板、白衣战士、蓝皮书等词语。这些词语中的 颜色语素都反映了该含色彩词语所指事物的颜色特 征。另一种是, 色彩语素间接参与含彩词语的意义构 成, 即其在含色彩词语的意义构成中起比喻、象征或 借代作用。如: 红人、白旗、黄道吉日、黑社会、看 清等等。这些词语没有出现颜色的本义。

1. 红

汉语中含 “红” 的颜色词语:

1. 红白喜事 : 男女结婚是喜事, 高寿的人病 逝的丧事叫喜事。有时也说红白事。泛指 婚丧。

2. 红榜 : 指光荣榜。

3. 红包: 包着钱的红纸包儿, 用于馈赠或 奖励等。

4. 红灯区 : 指某些城市中色情场所集中的地 区。

5. 红火 : 形容旺盛、兴隆、热闹。

6. 红利 : 指企业分给股东的利润或分给职 工的额外报酬; 参加集体生产单位的个人 所得的额外收益。

7. 红娘 : 媒人的代称。

8. 红旗 : 红色的棋子, 是无产阶级革命的 象征; 竟赛中用来鼓励胜者的红色旗子; 比喻先进。

9. 红人 : 称受宠信或重用的人; 成走红的 人。

10. 嗐红：迅速走红。

11. 红心 : 比喻忠于无产阶级革命事业的思 想。
12. 红运 : 好运气

13. 红装：妇女的红色装饰, 泛指妇女的艳 丽装束。；指青年妇女。

14. 满堂红：形容全面胜利或到处兴旺。

以上例子中，显示出在汉语里含 “红” 颜色词 语, 大都表示吉利、热烈、庆祝、重视、受欢迎等。 中国人对红色已有传统的概念, 在戏剧打扮红脸谱的 演员是演关胜、好人等角色。中国古代的建筑色彩主 要也用红色，显示高贵、富丽的感觉。总而言之，红 榜、红包、红股、红人、红运等词语在汉语中是与幸 福、快乐等意义相关联。

印尼语中 “红” 的象征意思有：火、停止、禁 止、刺激、革命、流血、暴力、勇敢、冲动。

下面是印尼语含 “红” 的颜色词语:

1. Si jago merah (红公鸡) : 是指火灾

2. Daerah merah (红地区) : 受到政强迫拆迁的 地区（政府的计划为了建公共设施）。

3. Angka merah (赤字) : 学生成绩单上的不及 格分数

4. Benang merah (红线) : 连接两件事情的关 系。

5. Langau merah (红牛虻) : 接受贿赂等谋取不 义之财的人。

6. Kartu merah (红牌) : 1. 最后的警告。2. 处 分, 处罚的, 因犯错误而下场 (一般是在运动 比赛）或被炒鱿鱼。

7. Perusahaan pelat merah (红牌子的公司): 国家的公司。

8. (Mendapatkan) lampu merah (受到红灯) : 被炒鱿鱼。; 没有得到许可。

以上由 “红” 构成的词语中, 最多是表示禁止 或停止的意思。如: 红地区、红牌、（受到）红灯、 赤字。对于 “红牌的公司” 是一种特殊情况, 因为在 印尼所属于国家的, 都用红色来形容。如: 公务员所 用的汽车或摩托车的号码牌是以红色为主, 因为都是 属于的国家的。

红色离不开革命的精神。中国和印尼同样使用 了红色作为国旗, 因为革命和勇敢、勇气联在一起。 红军、红旗、红心等都含有革命的意义。

在汉语和印尼语里有相似的词语像汉语的 “红 灯区”、“校样”与印尼语的 “红地区” 和 “赤字” ，具有不同的意思。

红色在巴厘岛的印度教里, 表示第二地位 (Kasta Ksatria)。是指贵族、军队、国家的领导 甚至总统或主席。

在汉语里 “红” 颜色几乎是代表集义词, 而在 印尼语中不都是。

2. 白

汉语中含 “白” 的颜色的词语 :

1. 白榜：对人进行处分或批评的布告。 
因多用白纸写, 所以叫白榜。

2. 白丁：封建社会里指没有功名的人。

3. 白璧微瑕 : 洁白的玉上面有些小斑点, 比 喻很好的人或事物有些小缺点。

4. 白璧无瑕 : 洁白的玉上面没有一点儿 小斑点, 比喻人或事物完美无缺。

5. 白领 : 指从事脑力劳动的职员。

6. 白色收入：指按规定获得的工资、津贴等 劳动报酬, 具有公开性。

7. 白色恐怖 : 指在反动政权统治下, 反革 命暴力所造成的恐怖, 如规模的屠杀、逮 捕等。

8. 白事：指丧事。

9. 白眼 : 眼睛朝上或向旁边看, 是看不起 人的一种表情。

10. 白眼儿狼 : 比喻忘恩负义的人。

11. 白纸黑字：白纸上写的黑子, 指见于书面 的确丵的证据。

12. 白字：写错或读错的字; 别字。

“白” 颜色在汉语有丰富又有矛盾的意思。一 方面是与红色有相反的意思一反动、悲哀, 如: 白 榜、白丁、白事、白灾, 甚至有 “白色恐怖” 的名 称。这些词语和死亡、没有功名还有一些不吉利的事 情几乎都连在一起。

汉语的 “白” 颜色和哀悼、死亡有密切的关 系, 但是从那件事情我们也会找到一种深刻的感情, 就是思念和孝顺。因为在葬礼时, 穿白色的衣服也叫 白色孝服。葬礼是哀悼的日子, 穿着最朴素、最简单 的衣服来表明对亲人死亡的悲痛。

另一方面是含有纯洁、清白、正当、合法的象 征意义。像白璧无瑕、白色输入是褒义词。这一方面 的意思和印尼语具有相同的概念。

印尼语里含有 “白” 的词语:

1. Anak putih（白孩子）：是指兄弟姐妹的 第七个孩子。

2. Baju putih (白衣) : 老百姓。

3. Benang putih (白线) : 单纯; 纯洁。

4. Bubuk putih (白粉) : 是指吗啡。

5. Hidung putih (白鼻子) : 色狼。

6. Darah putih (白血) : 贵族的血统 (与 “ 蓝血”的意思相同）。

7. Mobil putih (白车) : 救护车。

8. Putih hati (白心) : 诚实; 乐意助人。

9. Berputih tulang (白骨头) : 去世; 死 亡。

10. Memutihkan mata（使眼睛变白）：使感到 差愧。；刺痛人心

印尼语的“白”颜色主要是表示忠诚、诚实的 意思, 像benang putih (白线) 、putih hati (白 心）。此外, 印尼的国旗是红白色。因为印尼人对
白色的忠诚、纯洁和洁白有深刻的概念。印尼的国花 是茉莉花, 也是代表真诚的意思。不奇怪在印尼, 送 别人 “红包” 时, 是用 “白包” , 是表示诚心诚意的 心意。

无论是汉语还是印尼语, 白色与善事也有联想 意义。如: 白衣天使、白衣战士、mobil putih（白 车)。

另外, 印度教认为白色是印度教里面最高的地 位, 指知识分子、印度教的牧师。他们相信那些人是 神圣人, 因此使用了白色来代表。

当然, 在印尼语白色也有贬义, 可在印尼人心 目中白色就是忠诚、真诚的代表。

3. 黄

汉语中含 “黄” 的颜色词语：

1. 黄包车 : 人力车

2. 黄道吉日 : 迷信的人认为宣于办事的好日 子。

3. 黄毒 : 指毒害人思想的淫秽的书刊、音 像制品等。

4. 黄花 : 除了是指菊花以外, 还指没有过 性的行为的（青年男女）。

5. 黄口小儿 : 指婴儿、多用来讱诮无知的年 轻人。

6. 黄片：指内容淫秽的影视片。

7. 贩黄 : 贩卖黄色书刊、录像带、光盘 等。

印尼语包含 “黄” 的颜色的词语:

1. Korps baju kuning (穿着黄衣服) : 卫生 员

2. Bendera kuning（黄旗）：表示哀悼

3. Buku kuning (黄书) : 廉价小说。; ( 印尼古兰经学馆采用的）伊斯兰教教义课 本。

4. Kartu kuning (黄卡) : 部门发出给求职 者的填表, 内容是求职者简介、学历等说 明。

5. Koran kuning（黄色报纸）：写关于明星 闲话的报纸

6. Lampu kuning（黄灯）：表示警告

7. Semangat bambu kuning (龙头竹精神) : 争取国家, 勇敢面对敌人的精神, 爱国之 心。

8. Sirih kuning (黄色的萎叶) : 比喻漂亮 的姑娘

9. Pantat kuning (黄色屁股) : 比喻小气的 人

黄色是中国人的象征之一。外国人一听 “黄 河” 就知道是指中国。中国人的皮肤属于黄皮肤, 土 地也是黄土地。可见, 黄色与中国人有密切的关系。 汉民族过去的皇帝所有的事情几乎都会与黄色有关, 
如：“黄袍” 是天子的 “龙袍”、“黄钱” 是天子 的 “依仗”、“黄榜” 是天子的诏书等等。因而, 黄 色就成为贵族的颜色——皇帝, 所以表示高贵、尊 贵、贵族的颜色。

在印尼黄色也成为贵族的代表颜色, 只是不像 在中国那么普遍。在几个地方像日惹和梭罗还保留 “ 宫殿和皇帝” 的形象。他们在举行活动时主要是穿着 金灿灿的衣服，表示他们的地位比一般人高一些。

除了高贵以外，印尼人视黄色为吉祥和庄严的 象征。在举行主要活动时, 印尼人一般会准备 “黄 饭”。不仅是成为礼饭, 而且表示吉利、顺利完成一 切。

印尼语中的 “semangat bambu kuning (龙头 竹精神) ”, 是一种表示激烈的精神。当印尼成为别 的国家的殖民地时, 印尼人没有什么高级的武器, 老 百姓就以竹枪作为武器。带着简单的武器和巨大的勇 气最后打败了殖民者。

黄色最常用是表示警告, 像 “lampu kuning（ 黄灯）”。在运动或比赛我们也会常用 “黄牌”, 意 思就是给运动员或教练员出示的黄色警示牌。可, 在 印尼语 “kartu kuning” 还有另外意思, 即政府的规 定给求职者在求职时必须填的表格。

另外，黄色在巴厘岛印度教里面是指第三地位 的人。他们主要是在国家从事经济事业人, 老板、会 计人员等有关经济方面的事业。

随着社会的发展, 黄色的意义在汉语中也发生 了变化。以上的例子说明了在现代汉语, 黄色已经含 有贬义一一表示色情的颜色。“黄” 颜色在汉语是很 特别的颜色, 从传统包含了襄义的颜色至现代变成贬 义的颜色, 是一种很大的差异。

4. 蓝

汉语中含 “蓝” 的颜色词语:

1. 蓝领 : 某些 国家或地区指从事体力劳动 的工人，他们劳动时一般穿蓝色工作服。

2. 蓝青官话 : 方言地区的人说的普通话, 夹杂着方音。

3. 蓝色农业：指进海水产养殖业, 因海水的 蓝色的, 所以叫蓝色农业。

4. 蓝图：用感光后变成蓝色（或其他颜 色）的感光纸制成的图纸。; 比喻建设计 划。

印尼语包含 “蓝” 颜色词语:

1. Baju biru (蓝衣) : 犯人在监狱穿的衣 服。

2. Darah biru（蓝血）: 贵族的血统

3. Film biru（蓝色电影）：色情片或者黄色 片。

4. Buku biru (蓝本) : 社会名人录。; 写上 对某件事情的意见、建议的书。; 官方报 告
蓝色使人们想到蓝色的天空和大海, 给内心平 静的感觉。汉语中, “蓝” 往往用 “青” 来表示。 如, “青天”、“青云” 是天空蓝天的意思。所谓大 海的颜色, “蓝色农业” 来称呼在海水工厂或工作 人。

而蓝色在印尼语中除了表示天空的颜色, 印尼 语蓝色多指高贵、贵族的血统。同时也包含了贬义, 就是指色情的事情或东西。这一点是与汉语中黄色的 意义相同。

5. 绿

汉语中含 “绿” 的颜色词语:

1. 绿地：指城镇中经过绿化的空地。

2. 绿肥 : 把植物的嫩茎叶反压在地理, 经 过发酵分解而成的肥料。

3. 绿肺: 比喻能吸收二氧化碳并释放出氧 气的绿地、森林等。

4. 绿帽子 (绿头巾) : 称妻子 有外遇为戴绿 帽子。

5. 绿色壁垒：指为了保护本国或本地区环境 和经济利益而附加的进出口贸易条件及限 制措施, 如提高进口产品质量标准或实行 高额征税等。

6. 绿色标志 : 环境标志

7. 绿色食品 : 指无公害、无污染的安全营养 型食品。

8. 绿色通道 : 指医疗、交通运输等部门设 置的手续简便、安全快捷的通道; 泛指简 便、安全、快捷的途径或渠道。

印尼语包含 “绿” 颜色的词语:

1. Baju hijau（绿衣）：军人或军队

2. Pupuk hijau (绿肥) : 把植物的嫩茎叶反 压在地理，经过发酵分解而成的肥料。

3. Masih hijau (还很嫩) : 是指经验还不多 的人，末成熟的。

4. Meja hijau (绿色桌子) : 法庭

5. Jalur hijau (绿通道) : 城市中的绿化地 带

6. Kodok hijau (青蛙) : 在中国常说 “收 保护费”，但不一样的是在中国是流讯干 的, 而在印尼则是由政府人员 (治安) 收。

7. Lalat hijau（绿苍蝇）：高利贷

8. Lampu hijau (绿灯) : 收到许可

9. Palu hijau (绿锤子) : 法官的决定

绿色联想到自然的和谐和宁静, 也给人们带来 了新鲜感。绿色在许多国家包括中国和印尼也成为军 队的服装颜色。不仅与自然有关, 而且与保护、安全 也有关系。

对于环保、健康等绿色联想意义, 汉语和印 尼语里没有太大的差别。如: 绿肥、绿色食品、 
开绿灯、绿色通道的说法。随着环境保护运动的开 展, “绿色” 更深入人心, 其作为 “生命” 象征的联 想意义已经在世界范围内取得了普遍的共识。

不过, 在汉语里绿色还表示卑微、贫贱。古代 的汉代以后, 服色成为人社会等级地位的代表。皇帝 穿着黄色服装、贵族穿着红色服装, 而学士及下级官 员穿着绿色服装。所以, 绿色就成为官卑职小、地位 低微的读书人的代称。还有, 在古典戏剧中悲剧的女 性是 “青衣” , 妓院被称为 “青楼”。至今, 在中国 对绿色还是有特别的想法就是对 “戴绿帽子” 或者 “ 绿帽子” 可以说是一种忌讳的说法。尤其是, 对男生 来讲, 意思就是妻子有外遇就是给丈夫戴上绿帽子。

印尼语中, 绿色是形容经验还不多的人或者 对某件事情不熟悉的人——masih hi jau”（还很 嫩）, 就像小孩那样那么幼稚和单纯。另外, 印尼语 里 最常用的说法是 “meja hijau（绿桌子）”，指 法庭的意思。

在印尼的一些地方, 绿色表示神秘、不可思议 的事情。举例子, 在日惹的南海 (Pantai Selatan) , 有个迷信就是去南海时, 不能穿着绿色的衣服。因 为绿色是南海皇后的服装。如果穿着绿色等于与皇后 奋斗。还有印尼人也有一种迷信是 “buto i jo” , 即 绿色的巨人。在传统故事, “buto i jo” 是又坏又可 怕的角色。虽如此, 至今印尼人还相信 “buto i jo” 的存在。

\section{6. 黑}

汉语中含 “黑” 的颜色词语：

1. 黑暗：比喻（社会状况）落后; （统治 势力) 腐败。

2. 黑白 : 比喻是非、善恶。

3. 黑帮 : 指社会上暗中活动的犯罪团伙其 他反动集团或其成员。

4. 黑车：指没有牌照的或非法运营的车 辆。

5. 黑地：指没有登记在国家地亩册子上的 田地。

6. 黑店 : 杀人劫货的客店（多见于早期白 话）。；指没有营业执照非法经商店、客 店等。

7. 黑户 : 指没有户口的住户。也指没有营 业执照的商号。

8. 黑话：帮会、流讯、盗匪等所使用的暗 语。; 指反动而隐晦的话。

9. 黑货：指漏税或违禁的货物。

10. 黑名单: 反动势力为进行政治迫害而开列 的革命者和进步人士的名单。；指有关部 门对不合格产品或违反规约的企业、个人 等开列的名单, 通过一定渠道向社会公 布。

11. 黑色收入：指通过贫污、受贿等非法手段 取得的收入。

12. 黑社会 : 指社会上进行犯罪活动及其他
非法活动的各种有组织的黑暗 势力, 如反 动帮会, 流讯、盗窃集团, 走私、贩毒团 伙等。

13. 黑市：暗中进行非法买卖的市场。

14. 黑窝：比喻坏人隐藏或干坏事的地方。

印尼语包含 “黑” 颜色的词语:

1. Anak hitam (黑孩子) : 兄弟姐妹的第八 个孩子

2. Awan hitam（黑云/阴天）：伤心、悲哀因 为某件事。

3. Belanda hitam（黑荷兰）：是指支持荷兰 队（印尼殖民）的当地人。

4. Benang hitam (黑线)：忌讳的事情

5. Buku hitam (黑本) : 坏人记录

6. Dunia hitam (黑世界) : 是指黑社会

7. Kambing hitam (黑羊)：替罪的人。

8. Ilmu hitam (邪恶法术) : 邪恶, 违反宗 教的法术

9. Jalan hitam（黑路）：违反的方法

黑色一一黑暗的颜色, 给人们带来了一种神秘 感, 严肃的感觉。在古代中国, 黑色是 “正色” , 作 为北方和冬天之色, 它象征着万物的归宿和终结。黑 色在封建社会时曾经是尊贵之色。我们知道中国的水 墨画和书法是中国文化的代表之一，水墨画只使用墨 水来画画、写字, 这推到了黑色为尊贵色。

黑色在印尼也被认为尊贵的颜色。印尼的巴迪 克多使用黑色或棕色为基本颜色。原因不仅因为要 搭配印尼人的皮肤, 最重要是黑色或棕色是尊重的颜 色, 穿起来时让人会有自豪感。印尼人对漂亮、可爱 的男或女孩一般用 “hitam manis”（黑里俏）的说 法来形容。

由以上的词汇, 无论在汉语或印尼语里面, 黑 色与 “白” 颜色相反的意义, 往往被人们联想到黑 暗、犯罪、非法, 等邪恶的意义。像黑社会、黑名 单、黑窝等词汇在汉语和印尼语具有差不多的概念。 印尼语中, 黑也是指法术 (不好的), 如: “i “ mu hitam” 、“dunia hitam”、“jalan hitam” 。“Belanda hitam (黑荷兰)” 的说法出现因为 印尼曾经被荷兰殖民。“黑”指背叛国家的印尼人。 当时有些当地人为了自己的利益, 而背叛了自己的国 家。印度尼西亚共和国成立以后, 就很少使用这个词 汇。

在印尼巴厘岛的印度教里面, 认为黑色是下低 级（老百姓）（Kasta Sudra）的颜色。老百姓靠身 体的力量来工作或生活, 他们的单纯、忠诚、听上 级的话。他们做具体的工作来帮助国家，像农夫、渔 夫、工人等等。

我们知道 “绿色食品” 指无害的食品, 而汉语 中还有 “黑色食品” 的说法。可是, 它是褒义词, 指 颜色呈黑色或黑褐色的食品, 营养价值相对较高的像 黑米、黑芝麻、木耳等等。 
我们的世界是五颜六色、多姿多彩的世界。大 自然就是颜色词的主要来源。因此, 在种种语言中, 颜色词的主要意义有客观性的意义。但是, 文化的联 想意义，就有不同的概念。

笔者在对比汉语和印尼语颜色下位词的过程中 发现，两种语言之间的基本颜色下位词具有异同。对 于一些形容词素的颜色, 汉语和印尼语似乎有相似的 意义。如, 表示深色或浅色的颜色。

因为不同国家的环境和情况、植物、动物等的 原因, 产生了不同颜色的下位词。如, 汉语里的 “雪 白”、“橄榄绿”、“乌黑”、“鹅黄”等。在印尼 语里没有相对应的词。相反如此。汉语里有重叠色彩 词, 像 “白茫茫”、“黑油油”、“黑沉沉” 等。这 类词, 印尼语没有。这是汉语颜色词的特点。这类 词, 表示强调的意思。而在印尼语中使用了深度比较 高的颜色词来形容。

另外，文化、语言和民族心理互相连接。从颜 色象征意义我们可以连接到国家的历史、经济、信教 等方面的关系。印尼语的颜色词大多与西方有相似的 概念, 原因是因为历史上印尼被殖民国家占了几百年 的历史（350年多的历史）。在这么长的时间或多或 少对印尼的文化带来了重要的影响。

印尼是以五个信仰为主, 每个信仰对每种颜色 有自己的观念。如, 基督教, 认为白色是纯洁的颜 色、伊斯兰教, 以绿色为主要颜色等其他信教。这对 颜色词象征意义也有关。

中国对颜色的观念从古代封建社会开始。古代 的中国人, 按照自然和皇帝来决定颜色的概念。因为 古代的中国人相信皇帝是 “天子”，或多或少皇帝对 中国人的思想也有影响。封建社会灭绝后, 中国向西 方国家开始开放起来, 从而就慢慢开始引进了西方国 家的文化。

以上原因, 产生了汉语和印尼语颜色象征文化 意义的异同。如红色和白色。对中国人和印尼人来 说, 都是很重要的颜色。中国的 “红白喜事”、印尼 的国旗都离不开这两种颜色。但是, 这两种颜色在两 个国家有较大的区别。印尼人认为, “白”颜色是表 示真诚、诚实。所以在婚礼时, 人们给的 “红包” 是 用白色的包来放现金。还有，对 “腐化芏落、色情的 东西或事情”，在现代汉语使用 “黄” 颜色来形容, 印尼语, 使用 “蓝”颜色来形容。

本文对汉印尼语基本颜色词的下位词对比, 发 现不同的自然环境影响到其下位词。另外, 历史、宗 教对民族心理也有密切关系, 因而对颜色词的文化象 征意义在不同民族与国家产生了不同的观念。
中国社会科学院语言研究所词典编辑室编. (2007). 现代汉语词典（第5版）。北京：商 务印书 馆.

Departemen Pendidikan Nasional. (2008). Kamus Besar Bahasa Indonesia Pusat Bahasa,

(Edisi Keempat). Jakarta: Gramedia Pustaka Utama.

Maman S. Mahayana. (1997). Kamus Ungkapan Bahasa Indonesia. Jakarta: Gramedia Widiasarana.

北京大学印度尼西亚语——汉语词典编写组. 印度尼 西亚语一一汉语、汉语一一印度尼西亚语实用 词典 (Kamus Praktis Indonesia - Tionghoa, Tionghoa - Indonesia). Jakarta: Dian Rakyat, 2004 (2).

解海江. (2004). 《章黎平. 汉英语颜色词对比研究》 . 上海: 上海辞书出版社.

李红. (2007). 《印. 现代汉语颜色词语义分析》. 北京: 商务印书馆.

叶军. (2001). 《现代汉语色彩词研究》.内蒙古: 内蒙古人民出版社.

梁敏和, 孔远志. (2002). 《印度尼西亚文化与社 会》. 北京: 北京大学出版社.

顾嘉祖, 陆昇, 郑立信. (2002). 《语言与文化》. 上海: 上海外语教育出版社.

汤平山, 许利平. (2005). 《印尼: 赤道上的翠绿》 . 香港: 香港城市大学出版社.

李尧. (2002). 汉语色彩词研究: 硕士学位论文. 南 京: 南京师范大学.

曹成龙. (2002). 汉语颜色词研究: 硕士学位论文. 黑龙江: 黑龙江大学.

于海飞. (2003). 色彩词研究: 硕士学位论文. 山 东: 曲华师范大学.

韩秋菊. (2006). 汉语成语色彩词研究: 硕士学位论 文. 山东: 山东大学.

黄有卿。（2006). 汉语颜色词的文化含义: 硕士学位 论文. 天津: 天津师范大学.

娄捷. (2008). 现代汉语基本颜色词词义研究: 硕士 学位论文. 山头: 山头大学.

杨蕾. (2009). 现代汉语颜色词之以知研究: 硕士学 位论文. 江苏: 扬州大学.

吴瑞明.（1994）. 印尼语的颜色词及其文化象征意 义. 解放军外语学院学报, 3(68), $40-46$.

王军. (1996). “颜色” 词语在英汉语言中意义上 的异同. 西安外国语学院学报, 17 (68), $79-82$.

陈启英.（1996）。汉语色彩词与汉民族精神文化. 云南民族学院学报, 4, 75-80.

邓克风. (2008). 谈颜色词的英汉文化内涵. 湖北 广播电视大学学报, 28(9), 105-106.

龚平. (2009). 含有颜色词汇习语的认知模型化分 析. 重庆科技学院学报, 1, $157-158$. 